\title{
A comparative analysis of data generated using two different target preparation methods for hybridization to high-density oligonucleotide microarrays
}

\author{
David Gold ${ }^{1}$, Kevin Coombes ${ }^{1}$, Dina Medhane ${ }^{2}$, Anitha Ramaswamy ${ }^{2}$, \\ Zhenlin $\mathrm{Ju}^{3}$, Louise Strong${ }^{4}$, Ja Seok $\mathrm{Koo}^{5}$ and Mini Kapoor*2
}

\begin{abstract}
Address: ${ }^{1}$ Department of Biostatistics, The University of Texas M.D. Anderson Cancer Center, Houston, Texas, USA, ${ }^{2}$ Murine Microarray \& Affymetrix Facility, The University of Texas M.D. Anderson Cancer Center, Houston, Texas, USA, ${ }^{3}$ Department of Molecular Genetics, The University of Texas M.D. Anderson Cancer Center, Houston, Texas, USA, ${ }^{4}$ Department of Clinical Cancer Genetics, The University of Texas M.D Anderson Cancer Center, Houston, Texas, USA and ${ }^{5}$ Thoracic Head \& Neck Medical Oncology, The University of Texas M.D. Anderson Cancer Center, Houston, Texas, USA

Email: David Gold - dgold@odin.mdacc.tmc.edu; Kevin Coombes - krc@odin.mdacc.tmc.edu; Dina Medhane -dmedhane@mail.mdanderson.org; Anitha Ramaswamy - aramasw@mail.mdanderson.org;

Zhenlin Ju - zju@mail.mdanderson.org; Louise Strong - lstrong@mail.mdanderson.org; Ja Seok Koo - jskoo@mail.mdanderson.org; Mini Kapoor* - mkapoor@mail.mdanderson.org

* Corresponding author
\end{abstract}

Published: 06 January 2004

BMC Genomics 2004, 5:2
Received: 02 October 2003

Accepted: 06 January 2004

This article is available from: http://www.biomedcentral.com/l47|-2/64/5/2

(C) 2004 Gold et al; licensee BioMed Central Ltd. This is an Open Access article: verbatim copying and redistribution of this article are permitted in all media for any purpose, provided this notice is preserved along with the article's original URL.

\begin{abstract}
Background: To generate specific transcript profiles, one must isolate homogenous cell populations using techniques that often yield small amounts of RNA, requiring researchers to employ RNA amplification methods. The data generated by using these methods must be extensively evaluated to determine any technique dependent distortion of the expression profiles.

Results: High-density oligonucleotide microarrays were used to perform experiments for comparing data generated by using two protocols, an in vitro transcription (IVT) protocol that requires $5 \mu \mathrm{g}$ of total RNA and a double in vitro transcription (dVT) protocol that requires $200 \mathrm{ng}$ of total RNA for target preparation from RNA samples extracted from a normal and a cancer cell line. In both cell lines, about 10\% more genes were detected with IVT than with dIVT. Genes were filtered to exclude those that were undetected on all arrays. Hierarchical clustering using the 9,482 genes that passed the filter showed that the variation attributable to biological differences between samples was greater than that introduced by differences in the protocols. We analyzed the behavior of these genes separately for each protocol by using a statistical model to estimate the posterior probability of various levels of fold change. At each level, more differentially expressed genes were detected with IVT than with dIVT. When we checked for genes that had a posterior probability greater than $99 \%$ of fold change greater than 2 , in data generated by IVT but not IIVT, more than $60 \%$ of these genes had posterior probabilities greater than $90 \%$ in data generated by dIVT. Both protocols identified the same functional gene categories to be differentially expressed. Differential expression of selected genes was confirmed using quantitative real-time PCR.

Conclusion: Using nanogram quantities on total RNA, the usage of dIVT protocol identified differentially expressed genes and functional categories consistent with those detected by the IVT protocol. There was a loss in sensitivity of about $10 \%$ when detecting differentially expressed genes using the dIVT protocol. However, the lower amount of RNA required for this protocol, as compared to the IVT protocol, renders this methodology a highly desirable one for biological systems where sample amounts are limiting.
\end{abstract}




\section{Background}

High throughput DNA microarray technology has proved to be a powerful approach for gene expression profiling in various cellular systems and has played a significant role in the molecular classification of cancer [1-5]. To generate a meaningful transcript profiling pattern specific to a desired cell type, it is essential to isolate a homogenous cell population using techniques such as cell sorting or laser capture microdissection [6]. However, such techniques yield low amounts of RNA, usually insufficient to perform DNA microarray experiments. In such cases, it is often necessary to employ RNA amplification methods to generate the microgram quantities of RNA required to perform these experiments. The use of RNA amplification methods warrants a thorough analysis and understanding of the variations introduced due to the methodology employed. It is vital to be able to distinguish between the real effects of the biological system being analyzed and changes introduced due to a difference in the methods used to generate the data.

Here we present a comparative analysis of the data generated using two different target preparation techniques for hybridization to high-density oligonucleotide microarrays (U95Av2 GeneChips, Affymetrix, Santa Clara, CA; [7]). We have performed transcription-profiling experiments with samples extracted from normal human tracheobronchial epithelial cells (NHTBE; Clonetics, San Diego, CA) and human pulmonary mucoepidermoid carcinoma cells (NCI-H292; American Type Culture Collection, Manassas, VA). These profiling experiments used U95Av2 GeneChips (Affymetrix, Santa Clara, CA) and two different methods for target preparation, namely, a standard protocol (involving in vitro transcription, IVT; $[7,8]$ ) and an amplification protocol (involving double in vitro transcription, dIVT; $[7,9])$. In the IVT protocol, 5-40 $\mu \mathrm{g}$ of total RNA is reverse-transcribed to generate cDNA using an oligo-dT primer containing the $\mathrm{T} 7$ promoter sequence. The cDNA is converted into double stranded DNA using random hexamers and transcribed in vitro in the presence of biotinylated ribonucleotides to generate biotin-labeled complementary RNA (cRNA). The cRNA is fragmented and hybridized to the arrays. The dIVT protocol, which requires only 50-250 ng of total RNA, is a modification of the IVT protocol where unlabeled cRNA is first synthesized followed by a second round of reverse transcription to generate cDNA and in vitro transcription to synthesize biotin-labeled cRNA.

Our results showed that the variation attributable to biological differences between samples was greater than that introduced by differences in the protocols used for target preparation. To identify differentially expressed genes between the two cell lines, we compared the posterior probability of fold change exceeding various thresholds in either protocol. When we checked for genes that had a posterior probability greater than $99 \%$ of fold change greater than 2 , in data generated by IVT but not dIVT, more than $60 \%$ of these genes had posterior probabilities greater than $90 \%$ in data generated by dIVT. Moreover, both protocols identified many genes in the same functional categories to be differentially expressed. Although the dIVT protocol is less sensitive than the IVT (a 10\% loss of detection in the number of genes whose expression levels could be measured), the requirement of much lesser amount of sample required for dIVT protocol (20-100 times less than IVT protocol), makes it a highly desirable methodology for target preparation, when sample amounts are limiting.

\section{Results and Discussion}

In order to be able to perform DNA microarray experiments using limited amounts of starting samples, several studies in the recent years have used amplification methods to generate sufficient RNA for these experiments. In these cases, it is vital to understand the relative effects of amplification methods on the results generated by these studies. The goal of this study is to perform a comparative analysis of the data generated using two different target preparation techniques for hybridization to high-density oligonucleotide microarrays (U95Av2 GeneChips, Affymetrix, Santa Clara, CA; [7]), an in vitro transcription (IVT; $[7,8]$ ) protocol that requires $5 \mu \mathrm{g}$ of total RNA and a double in vitro transcription amplification protocol (dIVT; $[7,9])$. Total RNA samples extracted from 2 cell lines, normal human tracheobronchial epithelial cells (NHTBE; Clonetics, San Diego, CA) and human pulmonary mucoepidermoid carcinoma cells (NCI-H292; American Type Culture Collection, Manassas, VA) were used for target preparation using IVT and dIVT protocols. The data set generated, consisted of 2 replicates per cell line per protocol used, resulting in a total of eight experiments that were used to investigate both cell type-based (normal and cancer) and protocol-based effect (IVT and dIVT) on the final results.

\section{Quality Control}

As an initial quality control measure to check for variations introduced by differences in target preparation, labeling, hybridization, and handling of individual samples, we compared the data reports for all the arrays as generated by MAS 5.0 [10]. The background levels and the range of percentage of probe sets called present (25-48\%) were within acceptable levels as described previously [10]. All the exogenously added prokaryotic hybridization controls showed signal intensities significantly above threshold limits [10] in all reports. Similar results were obtained for housekeeping genes such as GAPDH and $\beta$-actin, highlighting the efficiency and accuracy of target preparation and hybridization. 
Further quality control was performed at 4 levels (1) automated grid alignment of the probe cells, (2) spatial variation of probe cells, (3) probe outliers, and (4) distributions of the detection $p$-values. Automated grid alignment and spatial variation of probe cells were examined at the .CEL file level using MATLAB scripts [11]. Briefly, to assess grid alignment, we checked the alternating pattern of positive and negative control cells in the borders of each GeneChip. The borders of the chips consist of alternate probe cells containing oligonucleotide B2 (a DNA sequence complementary to this oligonucleotide is included in the hybridization cocktail) and oligonucleotide $\mathrm{B} 1$ that serve as positive and negative controls respectively (Affymetrix, Santa Clara, CA). The intensities for positive controls (blue) and negative controls (red) were plotted as a function of probe cell column position to determine the quality of grid alignment for each chip (Figure 2). Intersection of the spectra for positive (blue) and negative (red) control intensities would signify an alignment drift out of phase. Spatial variation in the probe cells was assessed by plotting the coefficient of variation of pixel-level intensities within each probe cell on a chip by position (Figure 3). This analysis is useful in detecting any spatial artifacts on the microarray. The overall quality of the arrays was assessed with dChip [12] to compute the percentage of array outliers and single outliers. For each array, array outliers measure the percent of probe sets with expression patterns that are inconsistent from the rest of the arrays. Single outliers are analogous for single probes, measuring the percent of probe outliers on each array (Table 1). In general, dChip flags an array when either single or array outlier percentages exceed 5\% and recommends removal of arrays from further analysis when any of these values is $15 \%$ or more. None of the chips were flagged based on these criteria. Finally, we looked at the percentage of probe sets on each array with detection $p$ values less than 0.01 or 0.05 (Table 1 ). Detection $p$-values are a measure of how likely a transcript was expressed at a level to be called present on the array. As a general rule, chips with less than $10 \%$ of the probe sets detected with $p$ $<0.01$ are excluded from further analysis. This rule was derived from empirical experience. All GeneChips in this study passed all four quality control checks.

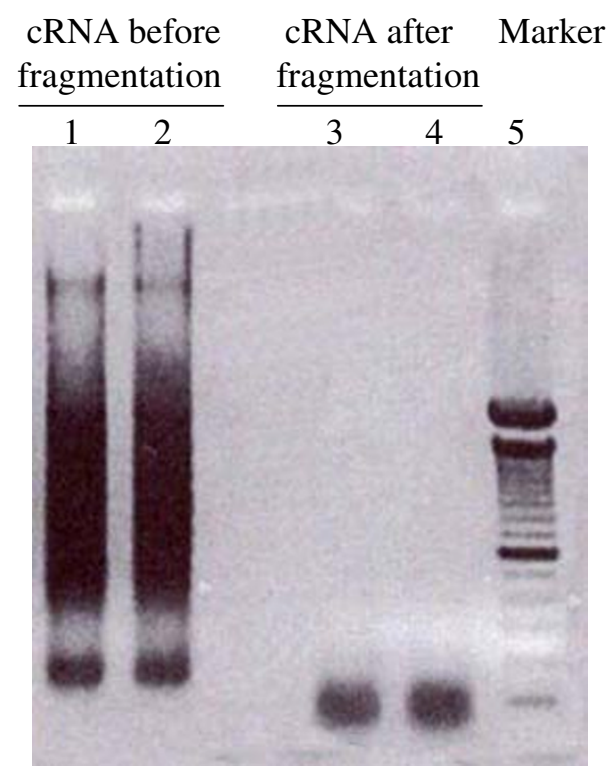

\section{Figure I}

Fragmentation of cRNA Aliquots of 2 cRNA samples were analyzed by agarose gel electrophoresis, before (lanes I and 2) and after (lanes 3 and 4) fragmentation. 


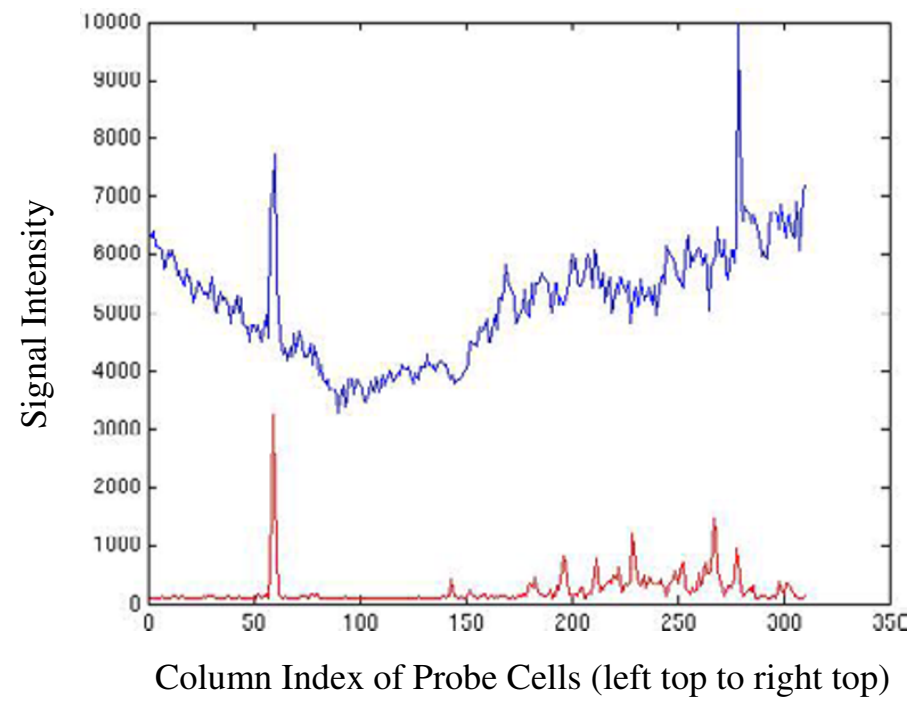

\section{Figure 2}

Grid alignment on microarrays. Plot of the intensity of positive controls (blue) and negative controls (red) from left to right across the top of the image, confirming that the scanner and quantification software were correctly aligned with the features on the microarray. The $x$-axis represents the column index from chip left top to right top corner. The $y$-axis represents the $75^{\text {th }}$ percentile of signal intensity of pixels in each probe cell.

\section{Comparison of the ability to detect genes using IVT and dIVT protocols}

The ability of the two protocols to detect expressed genes was examined using detection $p$-values. Because replicate chips were available, we had two measures of detection for each probe set in each group. We took as the detection $p$-value the maximum for each probe set over replicates as a conservative estimate of detection. The empirical distribution functions, which measure for any value $\mathrm{x}$ the percentage of values in the empirical sample less than or equal to $\mathrm{x}$, of detection $p$-values were plotted by protocol and sample type (Figure 4). Regardless of protocol, more probe sets were detected in cancer cells than in normal cells (compare figure $4 \mathrm{C}$ with $\mathrm{A}$ ). Almost all probe sets detected with the dIVT protocol were also detected (at the same $p$-value cutoff) with the IVT protocol (Figure $4 \mathrm{~B}$ and $4 \mathrm{D})$. In both cell lines, significantly more probe sets were detected (at a given cutoff of the detection $p$-value) using the IVT protocol than were detected using the dIVT protocol (Figure 4A and 4C). For example, at a detection cutoff of $p<0.01$ in the NCI-H292 cancer cell line, approximately $11 \%$ of the genes detected using IVT were not detected using dIVT (Figure 4C). At the same cutoff in the NHTBE cells, $8 \%$ of genes detected using IVT were not detected using dIVT (Figure 4A). At a detection cutoff of $p$ $<0.05$, the differences in detection rates were $12 \%$ and $10 \%$, respectively. Additional detection in both normal and cancer cell lines with dIVT was less than $2 \%$ at these levels. 


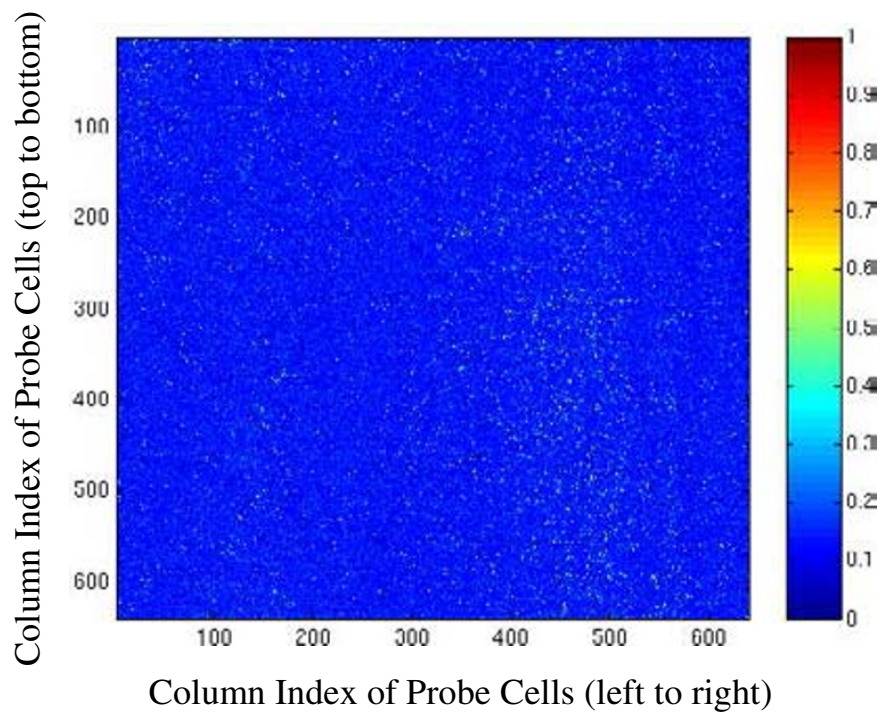

Figure 3

Spatial variation on microarrays Coefficient of variation of pixel-level intensities within each probe cell on a U95Av2 chip are plotted by probe cell position on the chip, confirming that there are no spatial artifacts on the microarray.

Table I: Quality metrics for 8 GeneChips using dChip (percentage of array outliers or single outliers) and MAS (percentage of probe sets with significant detection $p$-values)

\begin{tabular}{lcccc}
\hline Experiment & Array outliers (\%) & Single outliers (\%) & Detection P < 0.0 I (\%) & Detection P < 0.05 (\%) \\
\hline I. IVT, NHTBE & 0.02 & 0.04 & 21 & 31 \\
2. IVT, NHTBE & 0.06 & 0.16 & 24 & 34 \\
3. IVT, NCl-H292 & 0.05 & 0.04 & 35 & 45 \\
4. IVT, NCl-H292 & 0.23 & 0.09 & 22 & 47 \\
5. dIVT, NHTBE & 0.02 & 0.06 & 13 & 32 \\
6. dIVT, NHTBE & 0.40 & 0.20 & 25 & 20 \\
7. dIVT, NCl-H292 & 0.03 & 0.19 & 26 & 34 \\
8. dIVT, NCl-H292 & 0.04 & 0.10 & 36 \\
\hline
\end{tabular}

The decrease in the number of genes detected by dIVT is likely due to truncation of the 5 ' end of the RNA during amplification, as reported by McClintick and colleagues [15]. Luzzi and colleagues [8] found a similar decrease 
(A)

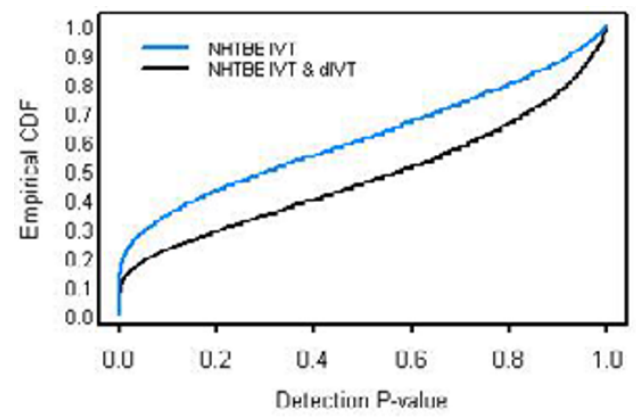

(C)

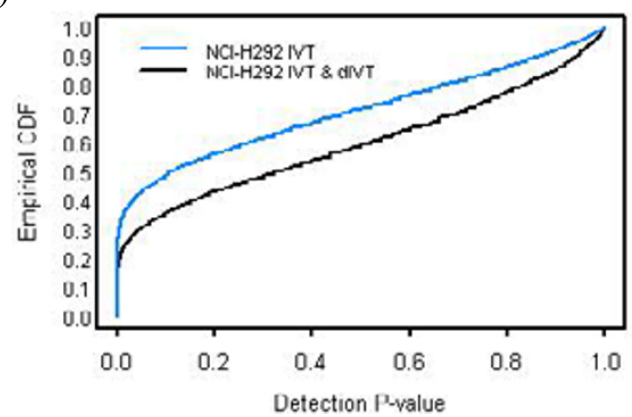

(B)

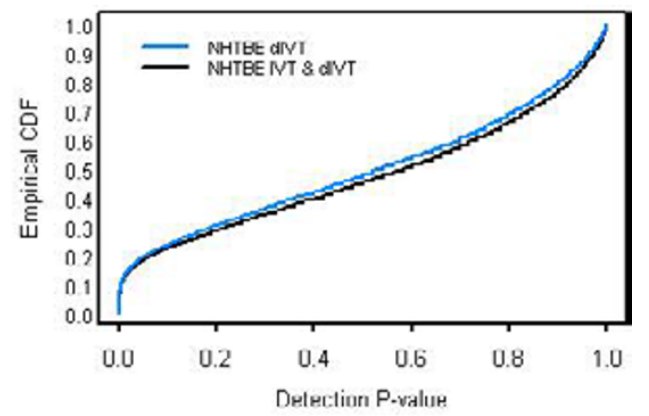

(D)

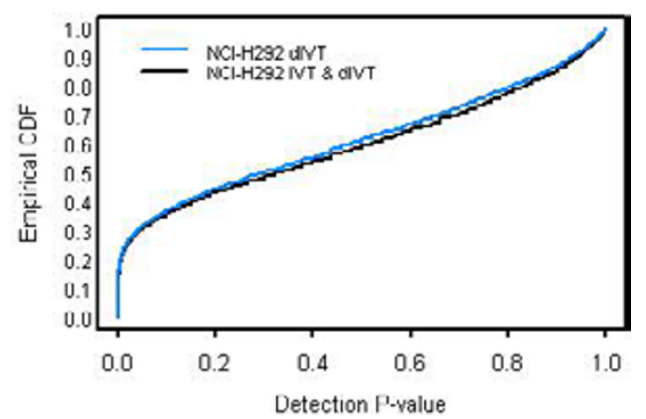

\section{Figure 4}

Empirical Distributions of the Detection $p$-values Detection $p$-values ( $x$-axis) are plotted against the empirical cumulative probability (y-axis) of values less than those on the corresponding curve. Plots shown in (A) and (B) represent data from normal cell line and those in (C) and (D) correspond to cancer cell line. The gap between curves in the plots shown in (A) and (C) corresponds to about 10\% of the genes that were detected using the IVT but not the dIVT protocol. The near coincidence of graphs in (B) and (D) shows that virtually all genes detected using the dIVT protocol are also detected using the IVT protocol.

$(11-16 \%)$ in the number of genes called present when using an amplification protocol for target preparation for hybridization to HuGeneFL microarray (Affymetrix, Santa Clara, CA). They also found that the overall hybridization signal, as measured by the scaling factor used for normalization in MAS 4.0, was approximately two-fold lower when using amplification.

By contrast, several studies have reported an increase in the number of detectable genes when combining amplification techniques with microarrays that use spotted cDNAs or long oligonucleotides on glass slides [16-19]. $\mathrm{Hu}$ and colleagues [16] suggested that the increase was due primarily to improvements in the signal intensity of low abundance genes, raising them above the noise level on the arrays. The difference in findings on the two different microarray platforms is probably related both to biochemical differences in the probe design and to differences in bioinformatical processing algorithms. According to a recently published thermodynamic model for estimating gene expression levels on oligonucleotide microarrays [20], it is conceivable that focusing on probes closer to the 3 ' end would find similar increases in the detection of low abundance transcripts. 
(A)

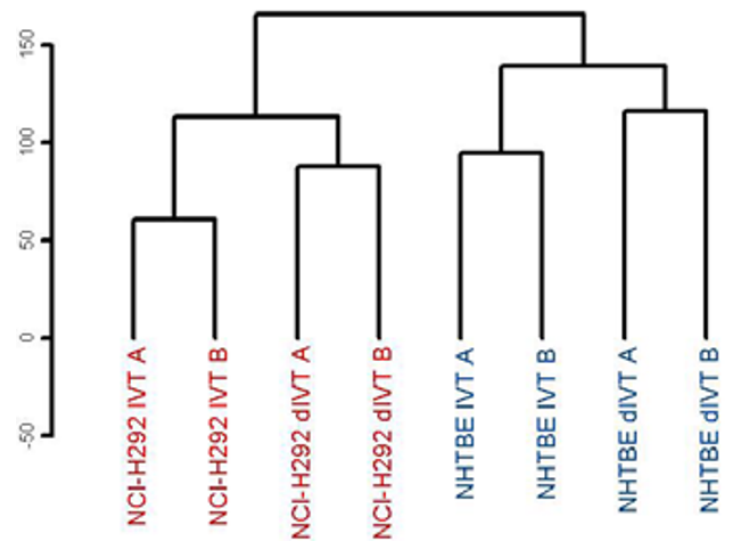

(B)

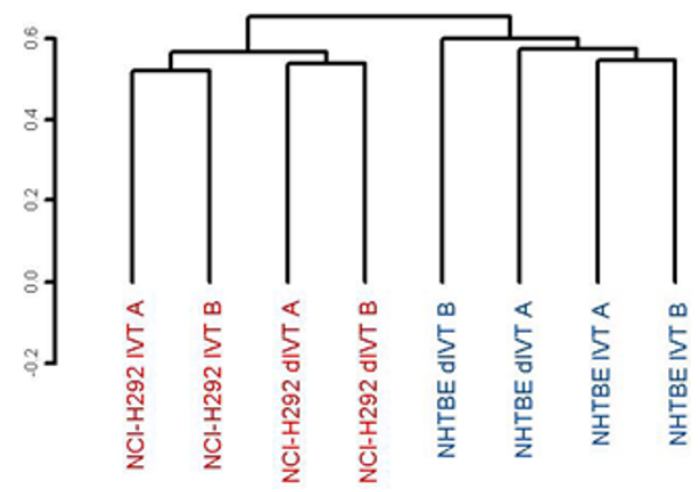

\section{Figure 5}

Hierarchical Clustering of samples Dendrograms of the samples based on the logarithmic expression values of 9,482 genes, using complete linkage and either (A) Euclidean distance or (B) Pearson correlation. Samples clustered by biological origin regardless of the target preparation protocol used. Suffix labels $A$ and $B$ denote replicates.

\section{Determination of overall variation in samples by hierarchical clustering}

Next, we used hierarchical clustering to explore the relative overall variation observed due to differences in sample source (cell lines) and protocol (IVT and dIVT) used for target preparation. The hierarchical clustering algorithm is agglomerative in that it joins samples based on a measure of multivariate distance, and once joined these samples are not free to cluster independently again. Pairwise joins in samples are represented as combined branches of a tree in a Dendrogram Plot (Figure 5). Since the results can be sensitive to the distance measure specified, both Euclidean and Pearson Correlation were used to determine if samples clustered together predominantly by protocol or biology. The probe sets were filtered based on detection $p$-values. For a probe set to be included in this analysis, the detection $p$-value had to be less than 0.3 on at least one chip. This choice was liberal enough to include most probe sets and to exclude the noisiest probe sets with the weakest signal. Of the 12,625 probe sets on the U95Av2 microarray, 9,482 passed this filter. We clustered samples by Euclidean distance between the logarithmic expression values of these 9,482 genes (Figure 5A). The major split in the clusters reflects the biological difference between cancer and normal samples, and the difference in protocols is a secondary effect. Within each group of samples, reproducibility (as measured by Euclidean distance between samples) was better with the IVT protocol than the dIVT protocol. Clustering based on the Pearson correlation between logarithmic expression values led to similar results (Figure 5B). These findings are consistent 
Table 2: Number of differentially expressed genes detected by each protocol as a function of the minimum fold change and the posterior probability of exceeding it

\begin{tabular}{|c|c|c|c|c|}
\hline Fold Change & Posterior Probability & IVT & dIVT & Both* \\
\hline \multirow[t]{4}{*}{1.5} & 0.99 & 430 & 311 & 116 \\
\hline & 0.95 & $14 \mid 1$ & 1122 & 513 \\
\hline & 0.90 & 2206 & 1737 & 852 \\
\hline & 0.85 & 2799 & 2234 & 1144 \\
\hline \multirow[t]{4}{*}{2} & 0.99 & 222 & 150 & 59 \\
\hline & 0.95 & 694 & 542 & 256 \\
\hline & 0.90 & 1073 & 883 & 428 \\
\hline & 0.85 & 1375 & 1145 & 551 \\
\hline \multirow[t]{4}{*}{4} & 0.99 & 76 & 50 & 19 \\
\hline & 0.95 & 207 & 155 & 90 \\
\hline & 0.90 & 298 & 249 & 142 \\
\hline & 0.85 & 375 & 314 & 179 \\
\hline \multirow[t]{4}{*}{8} & 0.99 & 33 & 21 & 6 \\
\hline & 0.95 & 90 & 69 & 44 \\
\hline & 0.90 & 129 & 97 & 65 \\
\hline & 0.85 & 152 & 121 & 79 \\
\hline
\end{tabular}

*number of genes observed to be differentially expressed by both protocols

with those from previous reports that used amplification protocols for target preparation for hybridization to microarrays $[21,22]$.

The biologic differences between the two human cell lines, normal tracheobronchial epithelial cells and pulmonary mucoepidermoid carcinoma cells, used in this study were expected to be quite significant. Hierarchical clustering showed that the variation between cell lines exceeded variation between protocols. Similar analysis performed using two more closely related samples may show larger relative variation by protocol.

\section{Comparison of differentially expressed genes detected by IVT and dIVT protocols}

The filtered dataset consisting of 9,482 genes (generated above) was used for comparing differentially expressed genes detected by IVT and dIVT protocols. Each of the 9,482 probe sets was modeled separately for each hybridization protocol using a linear model (see equations 1 and 2 below) to account for overall expression, cell line, and other variation on the logarithmic scale.

IVT Protocol $(5 \mu \mathrm{g}): \log _{2}\left(Y_{g i 1 k}\right)=\mu_{g 1}+\tau_{g i 1}+\varepsilon_{g i 1 k} \varepsilon_{g i 1 k} \sim \mathrm{N}(0$, $\left.\sigma^{2}{ }_{1}\right) \quad(1)$

DIVT Protocol $(0.2 \mu \mathrm{g}): \log _{2}\left(Y_{g i 2 k}\right)=\mu_{g 2}+\tau_{g i 2}+\varepsilon_{g i 2 k} \varepsilon_{g i 2 k} \sim$ $\mathrm{N}\left(0, \sigma_{2}^{2}\right)$

$g=1, \ldots, 12625$ probe sets, $i=1,2$ Cancer/Normal

$k=1,2$ Replicate Chips
The first term in each model, $\mu$, accounted for the overall log mean expression. The second term, $\tau$, accounted for differences between the normal cell line (NHTBE) and the cancer cell line (NCI-H292) on the log scale and represented fold change (FC) on the original scale. The final term, $\varepsilon$, accounted for unexplained variation and was modeled with a normal distribution on the log base 2 scale with mean 0 and variance $\sigma^{2}$. Since no prior information was available for these parameters, they were assumed to be generated a priori from uninformative prior distributions. The data were used to update our prior knowledge to produce posterior distributions [13]. These distributions were used to compute the posterior probability

$\mathrm{P}(F C<1 / \Delta \cup F C>\Delta \mid$ Data $)$

that $F C$ by cell line exceeded some desired amount $\Delta$. Hence, for a given $\Delta$, a high posterior probability in (3) is strong posterior evidence that the expected $F C$ lies outside of the interval $(1 / \Delta, \Delta)$. We examined these posterior probabilities for probe sets meeting the change criterion in each protocol for $F C>\Delta=1.5,2,4,8$ and probability cutoffs of $85 \%, 90 \%, 95 \%$ and $99 \%$ (Table 2). At each level of desired FC and probability cutoff, more differentially expressed probe sets were discovered with the IVT protocol than the dIVT protocol (compare columns 3 and 4 in table 2). This finding is consistent with the results presented above (Figure 4).

Figure 6A displays a Venn diagram comparing protocol performance for the criterion $\mathrm{P}(F C<1 / 2 \cup F C>2)>99 \%$ 
with the detection criterion implemented. Using these criteria 59 genes were found to be differentially expressed by both protocols, 163 found in IVT alone, and 91 in dIVT alone. The histograms in figures $6 \mathrm{~B}$ and $6 \mathrm{C}$ shows that nearly $60 \%$ of probe sets discovered with only one protocol still had posterior probabilities exceeding $90 \%$ in the other. For $F C=8$, about $31 \%$ of probe sets exceeded the top $93^{\text {rd }}$ rank in both protocols. Therefore we concluded that although fewer probe sets were discovered in the dIVT protocol, many were still detectable for differential expression at a lower threshold. This is a significant extension to the previous report by McClintick and coworkers [15] that relied on MAS probe set specific present/absent calls for detection analysis. They observed that genes at lower levels of detection were less reproducible in comparing treatment groups. We considered a range of cutoffs for detecting signal and change. We found that while reproducibility varied, for those genes showing at least $F C$ of 2 with $99 \%$ posterior probability in just one protocol, approximately $60 \%$ were detectable in the other for a slightly reduced posterior probability cutoff of $90 \%$ (Figure $6 \mathrm{~B}$ and $6 \mathrm{C}$ ).

\section{Comparison of functional gene categories identified as differentially expressed by IVT and dIVT protocols}

Since the two protocols did not identify identical sets of differentially expressed genes, we investigated whether the functional categories of the genes that were identified as differentially expressed were consistent. Each differentially expressed gene was annotated using functional annotations downloaded from the dChip web site, http:/ /biosun1.harvard.edu/complab/dchip/info file.htm. The number of differentially expressed genes detected by dIVT and IVT protocols in each functional category (with a posterior probability of 0.99 of having a fold change at least equal to 2) is shown in column 2 in tables 3 and 4 , respectively. These tables also show a count of a subset of these genes that were identified as differentially expressed by the alternate protocol for different cutoffs $(>0.90,>0.95$ and >0.99; columns 3, 4 and 5 in tables 3 and 4 ) on the posterior probability. In most functional categories, many genes detected with a posterior probability of 0.99 of having a fold change at least equal to 2 in one protocol were discovered at the slightly lower threshold of 0.90 in the other protocol (compare column 2 with column 3 in tables 3 and 4). These data show that the results generated by the two methods identify the same functional gene categories to be differentially expressed. Although the number of genes identified to be differentially expressed in each category by the two protocols may be different, the usage of any of these protocols will identify the same functional pathways to be affected when comparing the two cell lines.

\section{Confirmation by QRT-PCR}

Among the genes that were identified as differentially expressed by at least one protocol with a posterior probability of 0.99 of $F C$ exceeding 2, 10 were selected for confirmation studies using QRT-PCR. Differences in expression of these genes between the NHTBE and NCI$\mathrm{H} 292$ were estimated using the comparative $C_{T}$ method [14], normalizing the values relative to the housekeeping gene, GAPDH. Each gene was tested in duplicate reactions in three independent experiments. Replicates were used to compute $90 \%$ confidence intervals on the $\Delta \Delta C_{T}$ scale, which were then converted to confidence intervals on the FC scale (Table 5). For each gene, array FC model estimates are listed for IVT and dIVT protocols in columns 2 and 3, respectively. These are printed in bold by protocol for those meeting the change criterion. Notice that these show array FC estimates well exceeding 2 for their respective protocols. Four probesets were selected which met the change criterion in both protocols (TNF, K6A, SFP and IGFB), from the 59 in the set intersection in Figure 6A (center), two in IVT alone ( $\mathrm{LOH}$ and AXL) from the 163 in the exclusive IVT set (left), and four in dIVT alone (DMP, GR, EGFR and NFKB), from the 91 in the exclusive dIVT set (right). The following three columns list $F C$ with 90\% lower and upper bounds as modeled from the QRTPCR data. Down regulation is reported as 1/FC. Eight of the 10 differentially expressed genes tested, as determined by the microarray data using either protocol, produced similar results with QRT-PCR. SFP was observed to be up regulated by both array protocols and unchanged (QRTPCR Est. FC $=1 / 1.05$ ) by QRT-PCR. QRT-PCR results for EGFR showed down regulation while up on both array protocols. These contradictory results may be due to the presence of degenerate probe sets for these transcripts on the arrays that may cross hybridize with other genes, leading to false results. In a recently published report, out of a set of 66 genes identified as differentially expressed by macroarray screening, only 40 genes could be validated by QRT-PCR [23].

\section{Conclusion}

This study was performed to compare the relative effects of using two different protocols for target preparation for hybridization to high-density oligonucleotide arrays, on the final results generated by these experiments. Samples from two different human cell lines, a normal and a cancer cell line, were used for target preparation by the two different methods and hybridized to U95Av2 GeneChips. The conclusions derived from this study were that: (1) the decrease in the number of genes detected with the dIVT protocol was not substantial. For the 0.01 and 0.05 levels, the loss in detection for amplification was $8 \%$ and $10 \%$ respectively in the normal cell line. The loss was $11 \%$ and $12 \%$ in the cancer line (see Figure 4 ), (2) when comparing differentially expressed genes that had a posterior proba- 
(A)

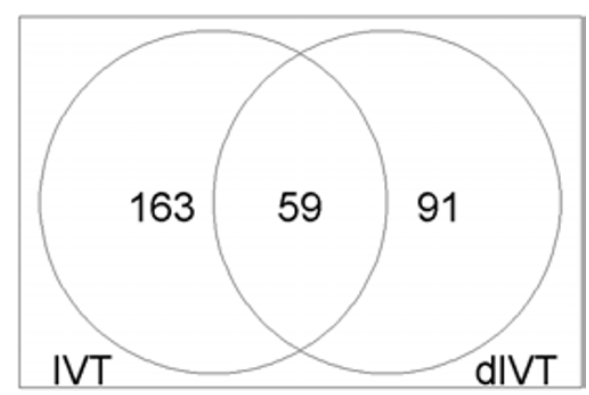

(B)

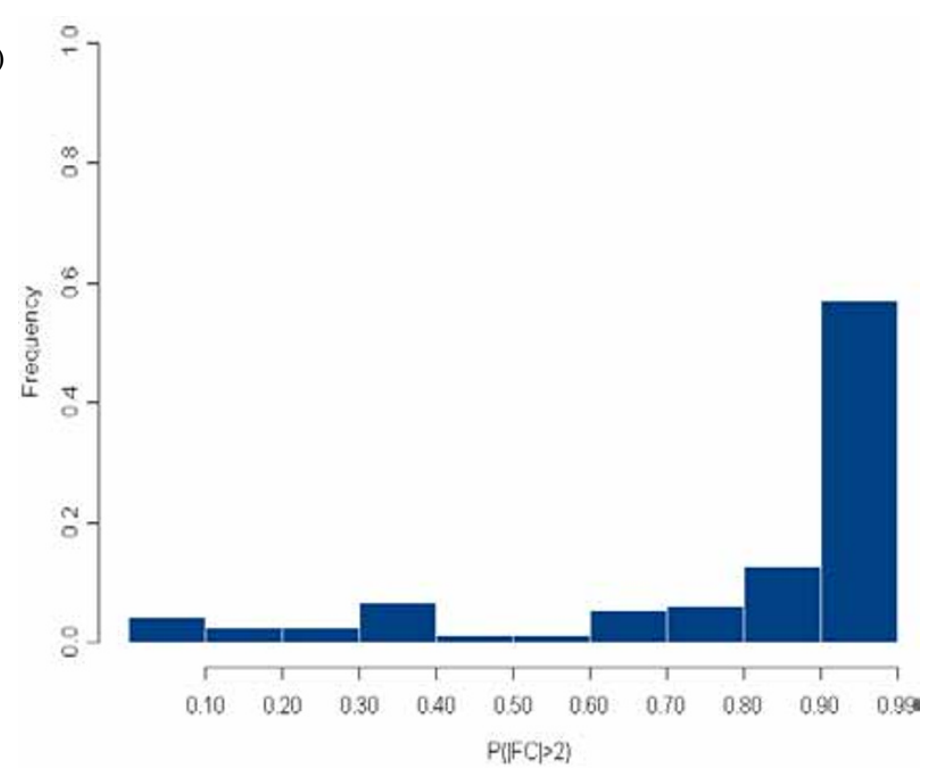

(C)

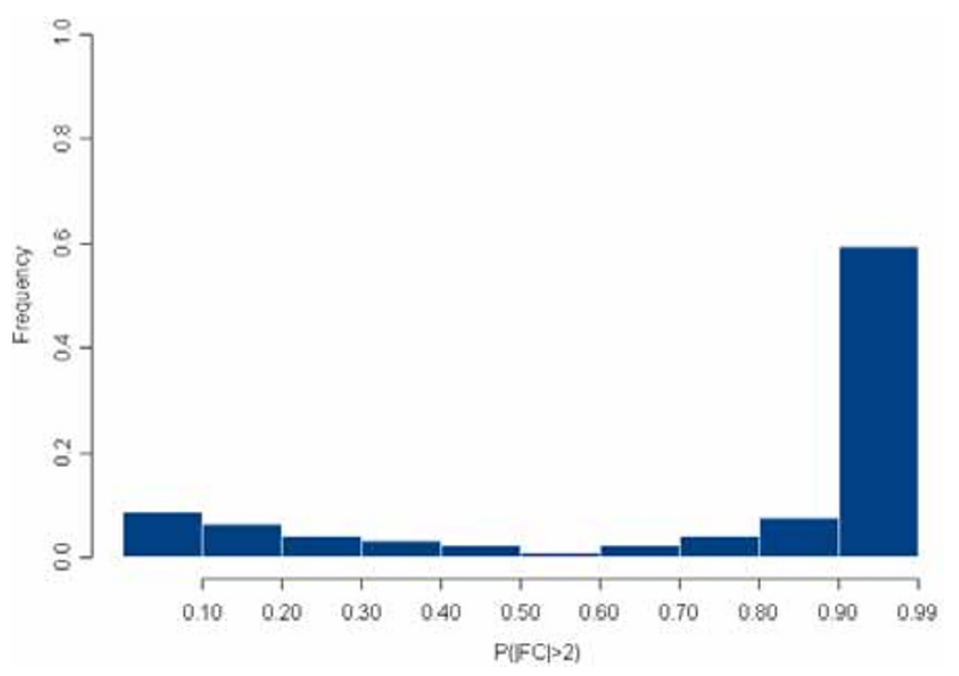

Figure 6

Comparison of differentially expressed genes detected by IVT and dIVT protocols (A) Venn diagram comparing genes with $\mathrm{P}(F C<\mathrm{I} / 2 \cup \mathrm{FC}>2)>99 \%$ shows 59 genes overlapped between the data generated by the 2 protocols. (B) Histogram of the posterior probability $\mathrm{P}(F C<\mathrm{I} / 2 \cup F C>2)$ in dIVT experiments for the 163 genes only called different using the IVT protocol. (C) Histogram of the posterior probability $\mathrm{P}(F C<\mathrm{I} / 2 \cup F C>2)$ in IVT experiments for the $9 \mathrm{I}$ genes only called different using the dIVT protocol. (A) and (B) show that more than $60 \%$ of the genes detected by only one protocol had posterior probabilities exceeding $90 \%$ in the other. 
Table 3: Detection of differentially expressed genes by dIVT Protocol for different functional gene categories

\begin{tabular}{|c|c|c|c|c|}
\hline \multirow{2}{*}{$\begin{array}{l}\text { Functional Category } \\
P(F C<1 / 2 \cup F C>2)\end{array}$} & \multirow{2}{*}{$\begin{array}{c}\text { Number of Genes } \\
\text { Detected by dIVT with } \\
>.99\end{array}$} & \multicolumn{3}{|c|}{ Number of Genes also Detected by IVT with } \\
\hline & & $>.90$ & $>.95$ & $>.99$ \\
\hline Apoptosis regulator & 0 & 0 & 0 & 0 \\
\hline Cell-cell signaling & 10 & 8 & 7 & 4 \\
\hline Cell cycle & 9 & 4 & 1 & 0 \\
\hline Cell death & 2 & 2 & 2 & 2 \\
\hline Cell motility & 5 & 5 & 5 & 0 \\
\hline Cell proliferation & 17 & 14 & 11 & 5 \\
\hline Defense and Immunity & 6 & 6 & 4 & 2 \\
\hline Extracelullar & 16 & 13 & 12 & 5 \\
\hline Homeostasis & 1 & 0 & 0 & 0 \\
\hline Nucleic acid binding & 19 & 7 & 4 & 3 \\
\hline Pathogenesis & 5 & 5 & 3 & $\mathrm{I}$ \\
\hline Signal transduction & 21 & 14 & 12 & 7 \\
\hline Stress response & 11 & 11 & 8 & 3 \\
\hline Transport & 5 & 3 & 3 & 2 \\
\hline
\end{tabular}

Table 4: Detection of differentially expressed genes by IVT Protocol for different functional gene categories

\begin{tabular}{|c|c|c|c|c|}
\hline \multirow{2}{*}{$\begin{array}{l}\text { Functional Category } \\
\mathrm{P}(F C<1 / 2 \cup F C>2)\end{array}$} & \multirow{2}{*}{$\begin{array}{c}\text { Number of Genes } \\
\text { Detected by IVT with } \\
>.99\end{array}$} & \multicolumn{3}{|c|}{ Number of Genes also Detected by dIVT with } \\
\hline & & $>.90$ & $>.95$ & $>.99$ \\
\hline Apoptosis regulator & 1 & 1 & 0 & 0 \\
\hline Cell-cell signaling & 9 & 7 & 7 & 4 \\
\hline Cell cycle & 6 & 3 & 2 & 0 \\
\hline Cell death & 3 & 2 & 1 & 1 \\
\hline Cell motility & 5 & 4 & 4 & 0 \\
\hline Cell proliferation & 13 & 10 & 8 & 5 \\
\hline Defense and Immunity & 5 & 5 & 5 & 2 \\
\hline Extracelullar & 19 & 14 & 11 & 5 \\
\hline Homeostasis & 0 & 0 & 0 & 0 \\
\hline Nucleic acid binding & 16 & 7 & 6 & 3 \\
\hline Pathogenesis & 6 & 2 & I & 1 \\
\hline Signal transduction & 27 & 18 & 13 & 7 \\
\hline Stress response & 15 & 13 & 12 & 3 \\
\hline Transport & 11 & 8 & 5 & 2 \\
\hline
\end{tabular}

bility greater than $99 \%$ of fold change greater than 2 , in data generated by IVT but not dIVT, more than $60 \%$ of these genes had posterior probabilities greater than $90 \%$ in data generated by dIVT. Thus, these genes may still have been discovered using dIVT protocol at a lower threshold based on a search, for instance that stressed functional stratification rather than simple criteria inclusion, (3) the two protocols showed reasonable agreement in the functional categories identified to be differentially expressed based on the gene lists generated by each protocol and (4) hierarchical clustering showed that variation due to biology of the samples was higher than that due to protocols used for target preparation.

\section{Methods \\ Cell Culture and RNA Extraction}

NHTBE cells (strain 6178, Clonetics, San Diego, CA) were seeded onto a $24 \mathrm{~mm}$ permeable membrane insert $(1 \times$ 
Table 5: Comparison of microarray and QRT-PCR estimates of FC for selected genes

\begin{tabular}{|c|c|c|c|c|c|}
\hline Gene & Est. Array FC* IVT & Est. Array FC dIVT & $\begin{array}{c}\text { QRT-PCR Lower } \\
90 \%\end{array}$ & QRT-PCR Est. FC & $\begin{array}{c}\text { QRT-PCR Upper } \\
90 \%\end{array}$ \\
\hline TNF & $1 / 19.53$ & I/I 53.58 & I/99.52 & $1 / 11.31$ & $1 / 1.29$ \\
\hline $\mathrm{K} 6 \mathrm{~A}$ & $1 / 16.2$ & I/I7.95 & $1 / 344.62$ & $1 / 56.69$ & $1 / 9.33$ \\
\hline SFP & 47.7 & 54 & $1 / 2.02$ & $1 / 1.05$ & 1.82 \\
\hline IGFB & 10.82 & 8.8 & 1.42 & 2.51 & 4.41 \\
\hline $\mathrm{LOH}$ & $\mid / 21.21$ & $1 / 5.66$ & $1 / 11.28$ & $1 / 3.61$ & $1 / 1.15$ \\
\hline$A X L$ & 36.82 & 13.02 & $|/| .21$ & 2.51 & 7.57 \\
\hline DMP & $1 / 18.94$ & I/29.04 & $\mathrm{I} / 55.24$ & $1 / 28.34$ & $\mathrm{I} / \mathrm{|} 4.54$ \\
\hline GR & $1 / 4.67$ & $1 / 20.49$ & $1 / 50.05$ & $1 / 10.37$ & $1 / 2.15$ \\
\hline EGFR & 4.58 & 6.27 & $\mathrm{I} / 37.44$ & $1 / 10.85$ & $1 / 3.15$ \\
\hline NFKB & 2.38 & 5.66 & 10.08 & 12.98 & $16.7 \mid$ \\
\hline
\end{tabular}

*Est. Array $F C$ in bold met criteria $\mathrm{P}(F C<1 / 2 \cup F C>2)>.99$.

$10^{5}$ cells/insert; Transwell-clear membranes, Costar, Cambridge, MA) and maintained in serum free BEGM media (Clonetics, San Diego, CA), containing insulin (5 $\mu \mathrm{g} / \mathrm{ml})$, transferrin $(10 \mu \mathrm{g} / \mathrm{ml})$, epinephrine $(0.5 \mu \mathrm{g} / \mathrm{ml})$, triiodothyronine $(6.5 \mathrm{ng} / \mathrm{ml})$, epidermal growth factor $(0.5 \mathrm{ng} / \mathrm{ml})$, bovine pituitary extract ( $1 \% \mathrm{vol} / \mathrm{vol})$, gentamicin $(50 \mu \mathrm{g} / \mathrm{ml})$, amphotericin B $(50 \mathrm{ng} / \mathrm{ml})$, and retinoic acid $(5 \times 10-8 \mathrm{M})$, until differentiated mucociliary bronchial epithelial cells were visible under a microscope. NCI-H292 cells were seeded at a density of $10^{6}$ cells per well in 6-well, flat-bottom, tissue culture plates (Corning, Corning, NY). Cultures were maintained in RPMI 1640 medium (Invitrogen Corporation, Carlsbad, CA) supplemented with 10\% fetal bovine serum (Invitrogen Corporation, Carlsbad, CA), penicillin (100 U/ml), and streptomycin $(100 \mathrm{~g} / \mathrm{ml})$. For RNA extraction, cells were washed twice in $1 \mathrm{x}$ PBS. The suspension was homogenized by 5-10 passages through a 20-gauge needle (0.9 $\mathrm{mm}$ diameter), passed through an RNeasy mini-column (RNeasy kit; Qiagen, Valencia, CA), and RNA was eluted in RNase-free water. RNA quantitation was performed by measuring absorbance of the RNA sample solutions at $260 \mathrm{~nm}$. The RNA integrity was determined by formaldehyde-agarose gel electrophoresis.

\section{Target Preparation}

For the standard protocol (IVT), $5 \mu \mathrm{g}$ of total RNA was reverse-transcribed in a $20 \mu$ l reaction with 200 U SuperScript II (Invitrogen Corporation, Carlsbad, CA) and 100 pmol of T7- $(\mathrm{dT})_{24}$ primer (5'-GGCCAGTGAATTGTAATACGACTCACTATAGGGAGGC GG-(dT)24-3') in 1X first-strand buffer (Invitrogen Corporation, Carlsbad, CA) at $42^{\circ} \mathrm{C}$ for $1 \mathrm{~h}$. The second-strand synthesis was performed at $16^{\circ} \mathrm{C}$ for $2 \mathrm{~h}$, in the presence of $E$. coli enzymes, DNA Polymerase I (40 U), DNA ligase (10 U), RNase H (2 $\mathrm{U}$ ) and $1 \mathrm{X}$ second-strand buffer (Invitrogen Corporation, Carlsbad, CA). The double-stranded cDNA was blunt- ended using $20 \mathrm{U}$ of T4 DNA polymerase, purified by phe$\mathrm{nol} /$ chloroform extraction and transcribed in the presence of biotin labeled-ribonucleotides, using the BioArray HighYield RNA transcript labeling kit (Enzo Laboratories, Farmingdale, NY) as described by the manufacturer. The biotin-labeled cRNA was purified using RNeasy mini-column (RNeasy kit; Qiagen, Valencia, CA) and fragmented at $94^{\circ} \mathrm{C}$ for $35 \mathrm{~min}$ in $1 \mathrm{X}$ fragmentation buffer $(40$ mMTris-acetate, pH 8.0, 100 mM KOAc, 30 mM MgOAc). For the dIVT protocol, the first and second cDNA strands were synthesized as described above. The first transcription was performed in the absence of biotin-labeled ribonucleotides, resulting in unlabeled cRNA, which was then used as starting material for the second cycle. In the second cycle, the first and second cDNA strands were synthesized as described above. The second transcription was performed in the presence of biotin-labeled-ribonucleotides, resulting in labeled cRNA. The cRNA was fragmented and checked by gel electrophoresis, as reported earlier [7]. A representative picture of such a gel is shown in figure 1 . The intact cRNA samples that appear as a smear on gels are reduced to $50-100$ bp fragments after treatment.

\section{Hybridization, Staining and Imaging}

The Affymetrix GeneChip system was used for hybridization, staining and imaging of the probe arrays. Hybridization cocktails of $300 \mu \mathrm{l}$ each containing $15 \mu \mathrm{g}$ of cRNA and exogenous hybridization controls were prepared as described previously [7] and hybridized to U95Av2 GeneChips (Affymetrix, Santa Clara, CA) overnight at $42^{\circ} \mathrm{C}$. Hybridized fragments were detected using streptavidinlinked to phycoerythrin (Molecular Probes, Eugene, OR; [7]). GeneChips were scanned and imaged using Affymetrix Microarray Analysis Suite (MAS), version 5.0 [10]. The MAS expression data report was generated for each sample and used to judge the quality of sample preparation and 
hybridization. The report includes information about noise, background and percentage of probe sets called present based on arbitrary threshold values [10]. Information about performance of exogenously added prokaryotic hybridization control genes such as $\mathrm{BioB}, \mathrm{BioC}$ and BioD [7] of the E. coli biotin synthesis pathway and the ratio of intensities of 3' probes to 5' probes for housekeeping genes such as GAPDH and $\beta$-actin are also included in the report.

\section{Microarray Data Analysis}

MAS, version 5.0, [10] was used for data acquisition, quantification, and normalization. This software is designed to take as input either the raw binary file containing the TIFF image pixel level information or a .CEL file, a smaller sized file summarizing the raw file. The software output includes summary statistics of probe set intensity such as signal and $p$-values for detection (detection $p$-values) testing whether or not signal was significantly greater than 0 . Quality of the automated grid alignment was assessed using a MATLAB (The MathWorks, Natick, MA) script [11]. These were developed to perform high-throughput quality control with Affymetrix GeneChips. Overall array quality was assessed using the MAS 5.0 detection $p$-values along with the outlier detection implemented in dChip [12]. Normalized data was exported from MAS 5.0 to S-Plus (Insightful Corp., Seattle WA), where the gene expression values were transformed by computing the logarithm (base two). We identified differentially expressed genes by constructing a linear model that accounted for known factors in the experimental design and applying Bayesian methods [13] to compute the posterior probability of a desired fold change $(F C)$.

\section{Quantitative Real Time PCR (QRT-PCR)}

Differentially expressed genes identified by microarray data analysis were validated by QRT-PCR. Four micrograms of template RNA were used in $210 \mu \mathrm{l}$ of RT reaction mix according to the manufacturer's instructions using the GeneAmp RNA PCR Core Kit (Applied Biosystems, Foster City, CA, USA). The reaction was performed at $42^{\circ} \mathrm{C}$ for $30 \mathrm{~min}$ followed by denaturation of enzymes at $95^{\circ} \mathrm{C}$ for $5 \mathrm{~min}$. Each QRT-PCR was performed in quadruplicate in $25 \mu \mathrm{l}$ volume in iCycler (Bio-Rad, Hercules, CA) using SYBR Green PCR Core Reagents (Applied Biosystems, Foster City, CA). Each PCR reaction used $0.2 \mathrm{mM}$ of each gene-specific primer (Invitrogen Corporation, Carlsbad, CA), $3 \mathrm{mM} \mathrm{MgCl}_{2}$, and $3 \mu \mathrm{l}$ of RT mix. GADPH was used as a reference. Delete sentence The thermo-cycling profile used was $55^{\circ} \mathrm{C}$ for $3 \mathrm{~min}, 95^{\circ} \mathrm{C}$ for $8 \mathrm{~min}$, and 40 cycles of denaturation at $95^{\circ} \mathrm{C}$ for $20 \mathrm{sec}$ and elongation at $60^{\circ} \mathrm{C}$ for $60 \mathrm{sec}$.

\section{QRT-PCR Data Analysis}

The QRT-PCR data were analyzed using the comparative CT method [14]. Briefly, the difference in cycle times, $\Delta \mathrm{CT}$, was determined as the difference between the tested gene and the reference housekeeping gene, GAPDH. We then obtained $\Delta \Delta \mathrm{CT}$ by finding the difference between treatments. The fold change was calculated as $F C=2^{-\Delta \Delta C_{T}}$. To determine confidence intervals, we used replicate measurements and experiments to model the variability in $\Delta \Delta \mathrm{CT}$ with a normal distribution. We then converted the intervals to the fold-change scale.

\section{Authors' Contributions}

DG and KC performed the statistical analysis, DM and AR carried out the target preparation and hybridization, ZJ and JSK performed the cell culture, RNA extraction and QRT-PCR, LS, KC and MK conceived the study and participated in its design and coordination. All authors read and approved the final manuscript.

\section{Acknowledgements}

This study was supported by NOI-CN-15I02 grant to LS.

\section{References}

I. Golub TR, Slonim DK, Tamayo P, Huard C, Gassenbeek M, Mesirov JP, Coller H, Loh ML, Downing JR, Caligiuri MA, Bloomfield CD, Lander ES: Molecular classification of cancer: class discovery and class prediction by gene expression monitoring. Science 1999, 286:53I-537.

2. Alizadeh AA, Eisen MB, Davis RE, Ma C, Lossos IS, Rosenwald A, Boldrick JC, Sabet H, Tran T, Yu X, Powell JI, Yang L, Marti GE, Moore T, Hudson J Jr, Lu L, Lewis DB, Tibshirani R, Sherlock G, Chan WC, Greiner TC, Weisenburger DD, Armitage JO, Warnke R, Levy R, Wilson W, Grever MR, Byrd JC, Botstein D, Brown PO, Staudt LM: Distinct types of diffuse large B-cell lymphoma identified by gene expression profiling. Nature 2000, 403:503-5II.

3. Bittner M, Meltzer P, Chen $Y$, Jiang $Y$, Seftor E, Hendrix M, Radmacher M, Simon R, Yakhini Z, Ben-Dor A, Sampas N, Daugherty E, Wang E, Marincola F, Gooden C, Lueders J, Glatfelter A, Pollock P, Carpten J, Gillanders E, Leja D, Dietrich K, Beaudry C, Berens M, Alberts D, Sondak V: Molecular classification of cutaneous malignant melanoma by gene expression profiling. Nature 2000, 406:536-540.

4. Perou CM, Sorlie T, Eisen MB, van de Rijn M, Jeffery SS, Rees CA, Pollack JR, Ross DT, Johnsen H, Akslen LA, Fluge O, Pergamenschikov A, Williams C, Zhu SX, Lonning PE, Borresen-Dale AL, Brown PO, Botstein D: Molecular portraits of human breast tumors. Nature 2000, 406:747-752.

5. Adorjan P, Distler J, Lipscher E, Model F, Muller J, Pelet C, Braun A, Florl AR, Gutig D, Grabs G, Howe A, Kursar M, Lesche R, Leu E, Lewin A, Maier S, Muller V, Otto T, Scholz C, Schulz WA, Seifert HH, Schwope I, Ziebarth H, Berlin K, Piepenbrock C, Olek A: Tumour class prediction and discovery by microarray-based DNA methylation analysis. Nucleic Acids Res 2002, 30:e21.

6. Luo L, Salunga RC, Guo H, Bittner A, Joy KC, Galindo JE, Xiao X, Rogers KE, Wan JS, Jackson MR, Erlander MG: Gene expression profiles of laser-captured adjacent neuronal sub-types. Nature Med 1999, 5: I 17-122.

7. Affymetrix: GeneChip ${ }^{\circledR}$. Expression Analysis Technical Manual Santa Clara, CA: Affymetrix; 200I.

8. Eberwine J, Yeh H, Miyashiro K, Cao Y, Nair S, Finnell R, Zettel M, Coleman P: Analysis of gene expression in single live neurons. Proc Natl Acad Sci 1992, 89:30 I0-30 I4.

9. Luzzi V, Holtschlag V, Watson MA: Expression profiling of ductal carcinoma in situ by laser capture microdissection and highdensity oligonucleotide arrays. Am J Pathol 200 I, I 58:2005-20I0. 
10. Affymetrix: Affymetrix Microarray Suite User's Guide, version 5.0. Santa Clara, CA; Affymetrix; 200I.

II. Baggerly KA: Detecting and correcting misalignment in Affymetrix data. 2002. The University of Texas M. D. Anderson Cancer Center, Department of Biostatistics Technical Report UTMDABTR-005-03. Available at: http://www.mdanderson.org/pdf/ biostats utmdabtr00503.pdf. Accessed August 14, 2003.

12. Li C, Wong WH: Model-based analysis of oligonucleotide arrays: Expression index computation and outlier detection. Proc Natl Acad Sci 200I, 98:3I-36.

13. Box GEP, Tiao GC: Bayesian Inference in Statistical Analysis New York: John Wiley and Sons; 1973.

14. Livak KJ, Schmittgen TD: Analysis of relative gene expression data using real-time quantitative PCR and the 2(-Delta Delta C(T)) Method. Methods 200I, 25:402-408.

15. McClintick JN, Jerome RE, Nicholson CR, Crabb DW, Edenberg HJ: Reproducibility of oligonucleotide arrays using small samples. BMC Genomics 2003, 4:4.

16. Hu L, Wang J, Baggerly K, Wang H, Fuller GN, Hamilton SR, Coombes $K R$, Zhang $W$ : Obtaining reliable information from minute amounts of RNA using cDNA microarrays. BMC Genomics 2002, 3:16.

17. Puskas LG, Zvara A, Hackler L Jr, Van Hummelen P: RNA amplification results in reproducible microarray data with slight ratio bias. Biotechniques 2002, 32: 1330-1340.

18. Nygaard V, Loland A, Holden M, Langaas M, Rue H, Liu F, Myklebost O, Fodstad O, Hovig E, Smith-Sorensen B: Effects of mRNA amplification on gene expression ratios in cDNA experiments estimated by analysis of variance. BMC Genomics 2003, 4: I I.

19. Wang J, Hu L, Hamilton SR, Coombes KR, Zhang W: RNA amplification strategies for cDNA microarray experiments. Biotechniques 2003, 34:394-400.

20. Zhang L, Miles MF, Aldape KD: A model of molecular interactions on short oligonucleotide microarrays. Nature Biotechnol 2003, $21: 8|8-82|$

21. Sotiriou C, Khanna C, Jazaeri AA, Petersen D, Liu ET: Core biopsies can be used to distinguish differences in expression profiling by cDNA microarrays. J Mol Diagn 2002, 4:30-36.

22. Dorris DR, Ramakrishnan R, Trakas D, Dudzik F, Belval R, Zhao C, Nguyen A, Domanus $M$, Mazumder A: A highly reproducible, linear, and automated sample preparation method for DNA microarrays. Genome Res 2002, I 2:976-984.

23. Calestani C, Rast JP, Davidson EH: Isolation of pigment cell specific genes in the sea urchin embryo by differential macroarray screening. Development 2003, 130:4587-96.
Publish with Bio Med Central and every scientist can read your work free of charge

"BioMed Central will be the most significant development for disseminating the results of biomedical research in our lifetime. "

Sir Paul Nurse, Cancer Research UK

Your research papers will be:

- available free of charge to the entire biomedical community

- peer reviewed and published immediately upon acceptance

- cited in PubMed and archived on PubMed Central

- yours - you keep the copyright

Submit your manuscript here:

http://www.biomedcentral.com/info/publishing_adv.asp
BioMedcentral 